\title{
PROUES DE NAVIRES DE PARIS
}

\author{
par M. Paul-Marie Duval
}

Par un rare privilège, il existe en France une grande salle romaine dont les voûtes sont intactes : c'est, au cœur du Musée de Cluny à Paris, la salle centrale $\mathrm{du}$ "Palais des Thermes". Ces voûtes retombaient sur des consoles en forme de proues de navires ${ }^{1}$. Ensemble doublement exceptionnel : les voûtes antiques encore en place sont si peu nombreuses que les consoles qui supportaient leur poids sont pour nous une rareté; les consoles en proues de navires sont, semble-t-il, uniques dans l'art antique.

L'étude de ces pierres sculptées, jusqu'ici négligées, permet de comprendre comment les architectes romains assuraient le soutien de leurs voûtes; leur présence à Paris nous invite à rechercher la place et le rôle que tiennent dans le legs artistique de Lutèce les décorations inspirées par l'élément liquide.

(1) Une seule avalt jusqu'à présent retenu l'attention des archéologues : Prosper Jollors, Mémoire sur les antiquités romaines et gailo-romaines de Paris, Mém. prés. par div. sav. à l'Acad. royale des Inscrip. et B. L., $2^{\theta}$ série (Antiquités de la France), t. I, 1843, p. 172 et pl. XXIII. - Albert Lenoir, Statistique Monumentale de Paris, Allas, t. I, 1867, pl.IV; Explication des planches (Coll. de doc. inédits sur l'Hist. de France), 1867, p. 7-8. - Émile EspérandieU, Recueil général des bas-reliefs, statues et bustes de la Gaule romaine, t. IV, $\mathrm{n}^{\circ} 3204$ (photogr. du moulage de la console $a$; mesures inexactes), $\mathrm{t}$. IX, 1925, p. 341. - F. G. de PAchtère, Paris à l'époque gallo-romaine (t. I de l'Hist. gén. de Paris), 1912, p. 86, n. 3, fig. 36. - Camille Jullian, Histoire de la Gaule, t. IV, 1914, p. 393, n. 5 ; t. V, 1920, p. 165 ; La console du Musée de Cluny, Rev. et. anc., XVI, 1914, p. 215-216; Le Paris des Romains (Coll. " Pour connaitre Paris", Hachette), 1924, p. 18; Au seuil de notre Histoire, I, 1930, 11/ leçon du cours au Collège de France (1905-1906), p. 243, n. 1-2.

C'est en 1946 que des échafaudages dressés pour l'entretien des voûtes m'ont permis d'étudier les consoles à loisir, d'en faire exécuter des photographies et des relevés géométraux, et de poser à nouveau le problème de leur interprétation : P.-M. Duval, Les proues de navires du a Palais des Thermes . de Paris, Comples rendus de l'Acad. des Inscrip. el B. L., 1946, p. 209-217, fig. 1-2; l'étude de la console $g$ est postérieure à cette communication oủ je ne signalais que quatre consoles conservées.

Que M. de Montrémy, conservateur du Musée de Cluny, M110 Élisa Maillard, assistante au Musée, et M. J: Trouvelot, architecte en che? des Monuments historiques, à qui je dois d'avoir pu établir cetto documentation, veuillent bien trouver ici mes vifs remerciements. Les dessins des fig. 5, 7, 10, 11, 13 et 15 sont dus à MN. Renard et Mastorakis, architectes en chef des Monuments historiques. 
La salle centrale du "Palais des Thermes » est rectangulaire, orientée sudest-nord-ouest dans le sens de la longueur; flanquée d'une piscine sur son côté nord, construite sur caves et située dans la moitié nord de l'édifice, elle ne peut être qu'un frigidarium. Quatre voûtes la recouvrent : voûte d'arête au centre, flanquée de deux berceaux à l'est et à l'ouest, un berceau perpendiculaire à ces derniers couvrant la piscine (fig. 1). Les consoles, taillées dans un calcaire

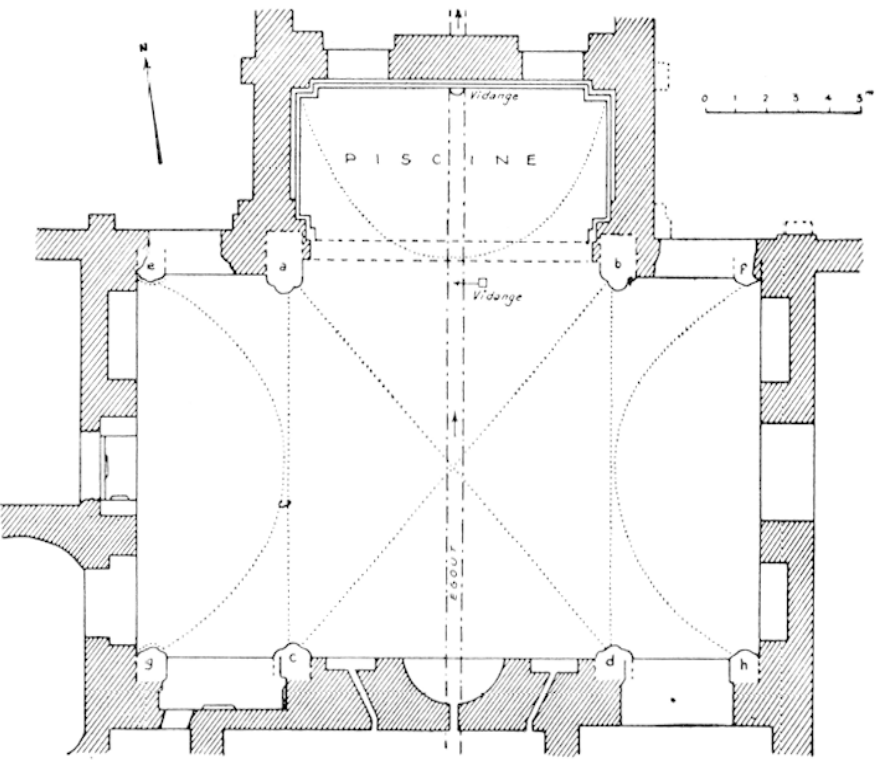

Fig. 1. - La grande salle du "Palais des Thermes" et l'emplacement des consoles $\mathbf{a}-\mathbf{h}$. assez grossier, situées à mi-hauteur $(7 \mathrm{~m}, 20)$ de la salle $\left(13^{\mathrm{m}}, 85\right)$, se répartissent ainsi : quatre d'entre elles (a, b, c, d) soutiennent les retombées de la voûte d'arête à leur jonction avec celles des berceaux latéraux; les quatre autres (e, $\mathbf{f}, \mathbf{g}, \mathbf{h})$, placées dans les angles, soutiennent les retombées extrêmes de ces berceaux. $\mathbf{a}$ et $\mathbf{b}$, situées aux angles de la salle et, de la piscine, sont plus longues que les autres, et leur long côté visible

est sculpté aussi bien que leur "ace; des autres, engagées sur trois de leurs côtés dans le mur, seule la face est sculptée et faite pour être vue (fig. 2) ${ }^{1}$.

(1) L'iconographhị du "Palais des Thermes * est riche d'œuvres originales depuis la Renaissance jusqu'à la création du Musée de Cluny (1844) : aussi longtemps que le monument fut une ruine laissée dans un pittoresque abandon. Une quarantaine d'cuvres d'art, souvent recopiées, ont été consacrées a la grande salle, mais rares sont celles qui reproduisent les consoles. Avant le xix ${ }^{\circ}$ siècle, celles-ci sont généralement escamotées ou remplacées par de simples blocs moulurès. Seul Hubert Robert a daigné les reproduire, en les restituant complètement, sur sa toile aujourd'hui au Musée Carnavalet (cf. E. DAcier, Le Tonnelier du Palais des Thermes, Soc. d'icon. parisienne, 3e année, 1910, Paris, 1911, p. 65-68, pl. XXXVII, qui date la toile des années 1780-1790). Sur sa toile ronde du Musée de Dijon (ácurie dans les ruines d'un ancien édifice.n, Catal., no 457, cf. Jullian, Le Paris des Romains, frontispice) comme sur la sanguine du Musée de Valence qui en est l'esquisse ("Vue des Thermes de Julien à Rome * [sic], cf. Dacier, o. c., pl. XXXVIII), la console a est représentée de façon informe. - Une gravure de Née, d'après J.-B.-F. Genillion, "Vue des restes du Palais des Thermes ", dans le Voyage pilloresque de la France, t. III, Paris, 1787, pl. no 56 ('f. JuI.LIAN, op. cit., pl. XI, dont la légende est intervertie 
Les quatre consoles centrales sont les plus larese : a : $1 \mathrm{~m}, 18 ; \mathbf{b}: 1^{\mathrm{m}}, 20$; c et $\mathbf{d}$ : il n'en reste rien, sauf la queue de $\mathbf{c}$ enfonce dans le mur, mais les encastrements sont bien conservés sur leurs bords et ont une largeur d'environ $1 \mathrm{~m}, 20$. Les quatre autres, e, $\mathbf{g}$ ef $\mathbf{h}$ (conserves), $\mathbf{f}$ (a en juger par l'encastrement), araient une largeur de 0 m, 90 . a et $\mathbf{b}$ sont plus saillantes que les consoles d'angles : $0^{\mathrm{m}}, 50$ contre $0^{\mathrm{m}}, 35$ en $\mathbf{h}$; il est vraisemblable que $\mathbf{c}$ et $\boldsymbol{d}$ étaient également plus saillantes, et que e, $\mathbf{f}, \mathbf{g}$ ne l'étaient pas plus que $\mathbf{h}$. La distribution des pierres était donc rationnelle au point de vue architectonique, leurs dimensions étant calculées en fonction du poids à supporter. Aussi bien, ce n'est point de chapiteaux qu'il s'agit, mais de consoles engagées dans le mur, ou corbeaux. Au point de vue esthétique, la position

avec celle de la pl. XIV; l'aquarelle originale de Genillion est au Cabinet des Estampes, coll. Destailleur, Dessins originaux sur Paris, vol. VI, n० 121, p. 134, n० 1266 du Catal. de la Collection), représentant le mur sud de la grande salle, montre qu'à cette date les con-

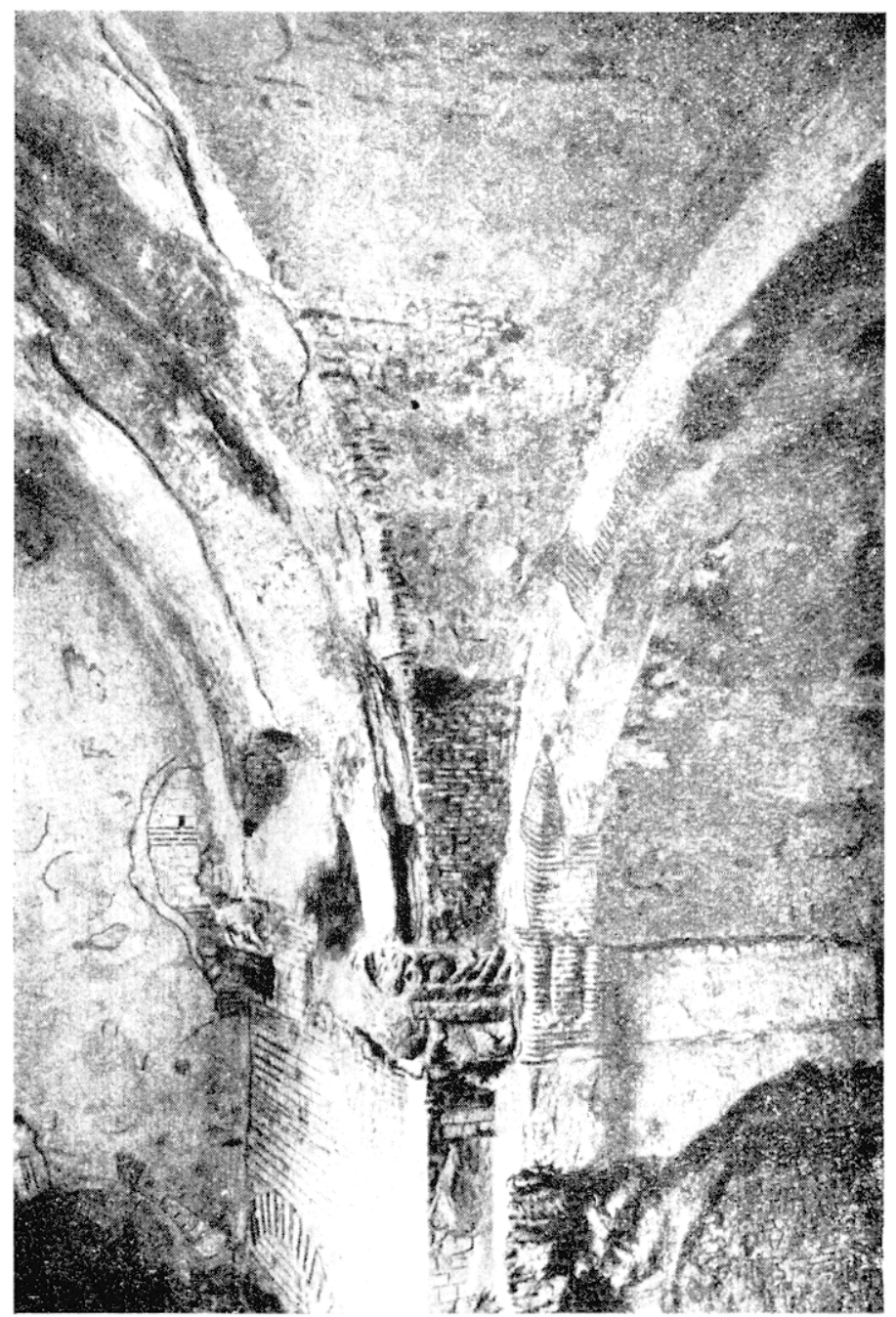

Fig. 2. - Situation des consoles : a (1 er plan), e (2e plan à gauche). soles c et d avaient déjà dis-

paru. - Les œuvres du XIX ${ }^{e}$ siècle montrent la console a dans son état actuel. La pl. XXIII de Jollois, o. c., donne face et profil, très inexactement; de même la pl. IV de Lenoir. $0 . c$. Uun dessin à vue assez exact est l'œuvre de l'architecte C. J. Bernard, Les Thermes de Lutèce, Magasin Pittoresque, LXXXII, 1914, p. 179-180. La seule œuvre d'art qui reproduise fidèlement les consoles a, b, c est une toile du Musée des Beaux-Arts de Poitiers due à J.-M.-Auguste Leroux (1901) représentant le mur nord et la piscine. Je remercie M. Charles Picard et M. M. Sandoz, conservateur des Musées de Poitiers, qui nous l'ont fait connaltre. 
des consoles d'angles était maladroite : les murs latéraux empêchaient le regard d'atteindre le bord sculpté qu'ils touchaient à son départ.

$\mathbf{a}, \mathbf{b}, \mathbf{e}, \mathbf{g}, \mathbf{h}$, seules conservées, représentent des proues de navires. Comme elles sont situées, les trois premières sur le mur nord, les deux dernières sur le mur sud, il serait invraisemblable que les trois autres - deux au sud, une au nord - n'aient pas eu la même forme. On peut donc affirmer que la décoration était homogène.
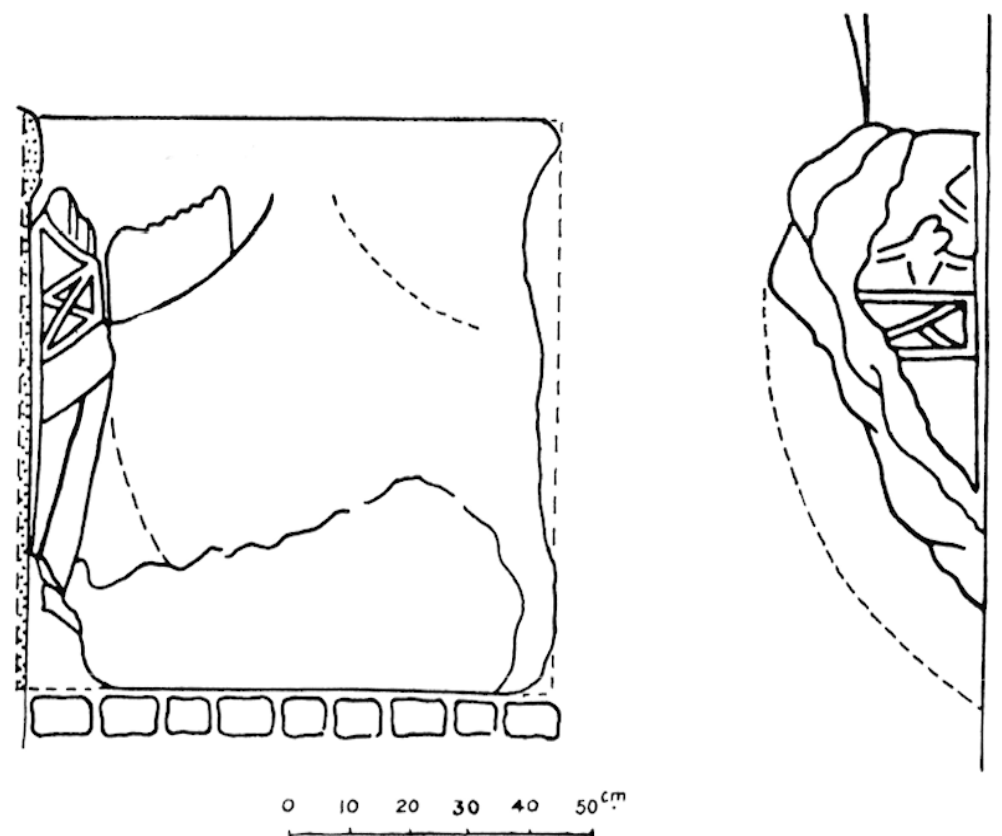

Fig. 3. - La console e, face et profil.

g est la plus mutilée. Mesurant $0^{\mathrm{m}}, 90$ au carré, elle est arasée, pour sa moitié gauche, au nu du mur. Sa moitié droite, fortement érodée, porte encore le profil d'une proue ventrue, qui n'est plus visible d'en bas.

e est, aussi très mutilée (fig. 3). De mêmes dimensions que $\mathbf{g}$, elle présente des traces de sculpture dans la moitié gauche et sur le bord de droite. A gauche : une rame en relief, cassée à son extrémité inférieure, s'écartant obliquement de la coque ; au-dessus, une préceinte très saillante surmontée d'un bastingage à croisillon sur lequel, à bord, est posé un objet indéterminé. La courbe concave de la coque montant vers le sommet de la proue, nettement visible, est surmontée d'un objet massif et renflé, tronqué à sa partie supérieure. - Sur le bord droit de la pierre, fait pour être vu de côté, on voit : au milieu de sa hauteur, un bastingage à croisillon en partie tronqué, surmontant l'amorce d'une préceinte; au-dessus, un objet impossible à définir.

h est moins dégradée (fig. 4). De mêmes dimensions, elle fait sur le mur une saillie de $0^{\mathrm{m}}, 35$. Sur sa face, le contour de la proue, large et ventrue, pointue à la base, est 
très net. A gauche, une rame en relief, tronquée à la base, se détache de la coque comme en $\mathbf{e}$; la préceinte a disparu, mais le profil du bastingage existe encore, surmonté d'un objet arrondi qui a tout l'air d'un bouclier. A droite, la face interne d'une rame oblique en relief, la préceinte et le bastingage sont encore visibles. Un objet arrondi reposait sur le bastingage. La partie supérieure de la proue, avec ses deux courbes concaves, est conservée : le sommet, cassé vers le haut, porte deux trous carrés superposés, profonds de 1 à $3 \mathrm{~cm}$., destinés au scellement d'un ornement de métal. Sur le flanc droit se voient les restes d'un torse humain en relief avec la tête et les bras; sur le flanc gauche,
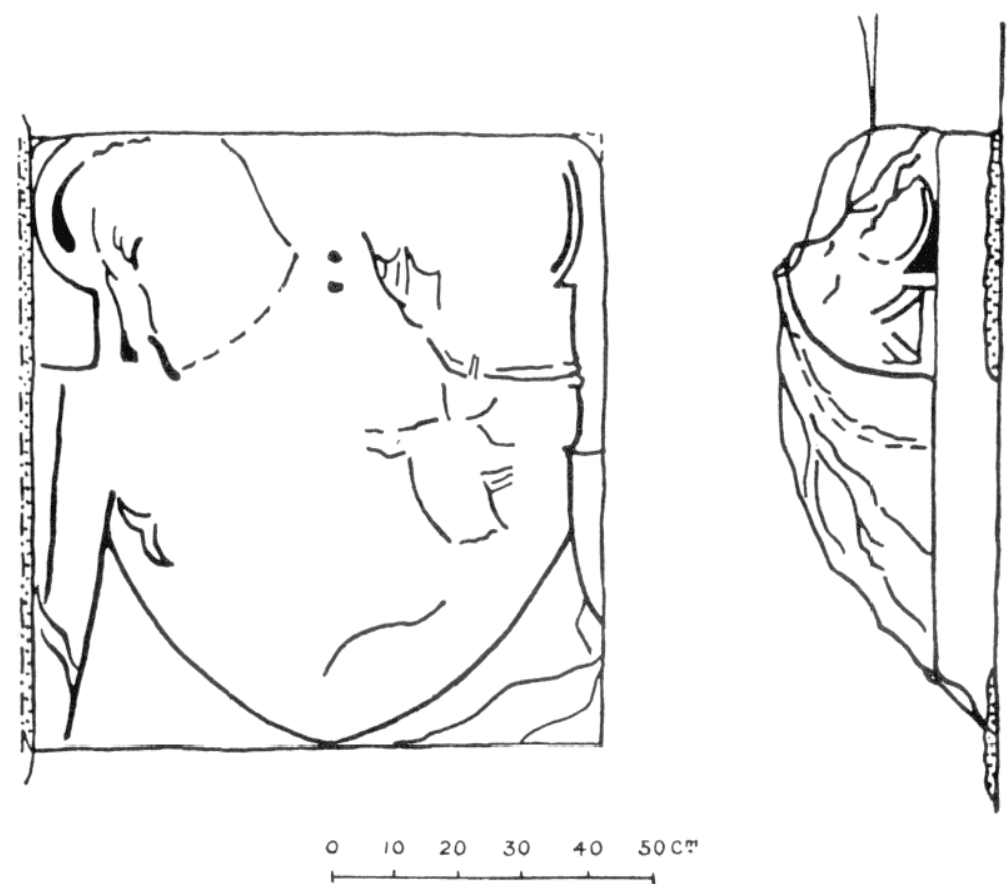

Fig. 4. - La console $h$, face et profil.

une queue de poisson : il devait y avoir un triton de chaque côté. Sur le bord droit, vu de côté, on retrouve la rame de droite, l'amorce de la préceinte, du bastingage avec croisillon, et de l'objet qui surmontait celui-ci et qui, de face, se présente bien comme un bouclier rond.

a est la mieux conservée, et la mieux connue. Mais toutes les descriptions qui en ont été données (cf. supra, p. 123, n. 1), sont vagues et incomplètes. Souvent dessinée, à vue, depuis le $\mathrm{xIX}^{\mathrm{e}}$ siècle, elle a fait l'objet, en 1906, sur l'initiative de Camille Jullian, d'un moulage qui se trouve au Musée des Antiquités Nationales à Saint-Germain-enLaye : c'est une photographie de ce moulage qu'a reproduite Espérandieu dans son Recueil. Mesurant, de face, $1^{\mathrm{m}}, 18$ de large sur $0^{\mathrm{m}}, 92$ de haut, elle a une longueur en queue de $1^{\mathrm{m}}, 87$, sculptée sur $1^{\mathrm{m}}, 12$ (fig. 5 ). Le relief de la proue fait une saillie de $0^{\mathrm{m}}, 50$ sur le mur, et la pierre n'est engagée dans celui-ci que sur les quatre cinquièmes de sa largeur, le dernier cinquième se trouvant en porte-à-faux vers l'intérieur de la piscine. Un pilastre 
en briques prolongé par un doubleau également en briques collé à la voûte à la ịonction de l'arête centrale et du berceau de la piscine pallie à cette maladresse : masquant en partie la queue de la pierre au delà de la sculpture latérale, il présente à cet endroit la forme d'un grossier triglyphe destiné probablement à recevoir un placage décoratif. L'extrémité de la queue est elle-même masquée par une banquette de maçonnerie reposant sur un arc, qui occupe le panneau ouest de la piscine à la base du berceau (fig. 2).

La face (fig. 6-7) a

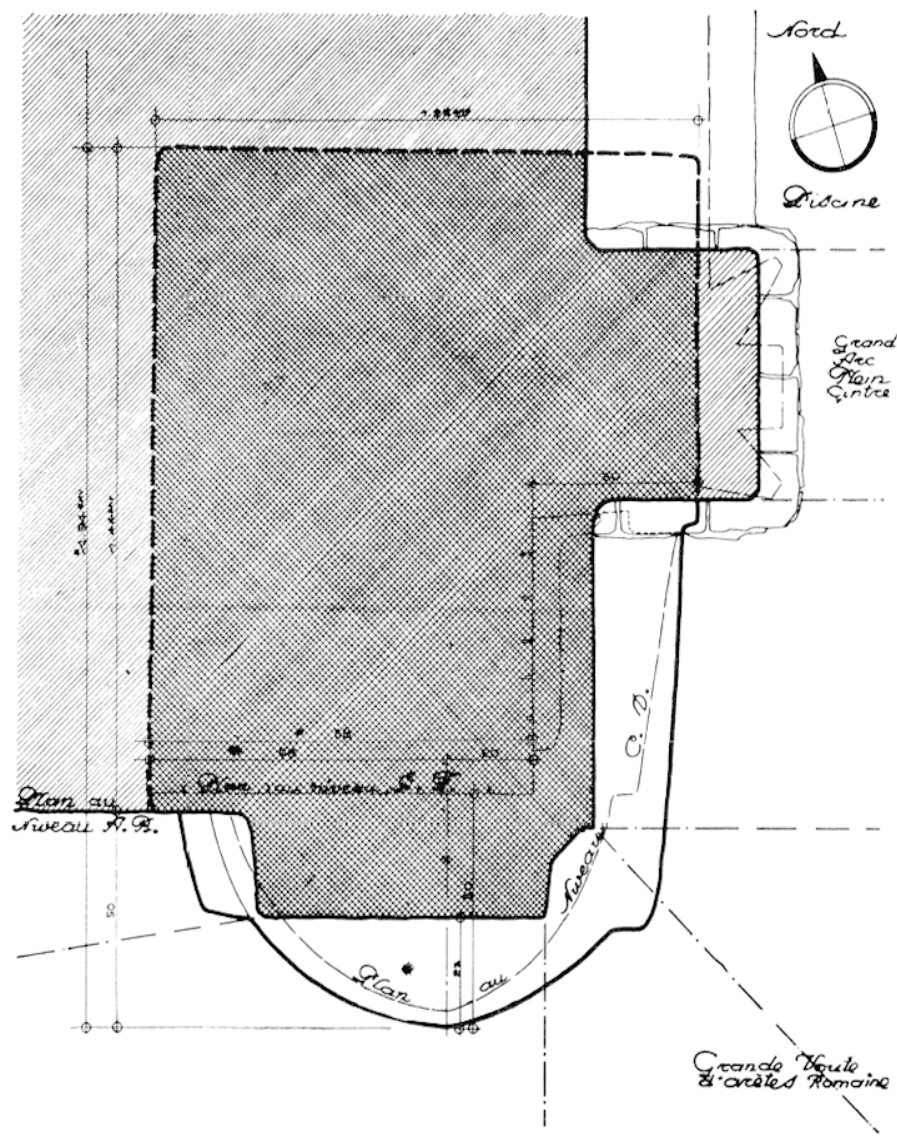

Fig. 5. - Plan de la console a. la forme d'une proue ventrue analogue aux précédentes et décorée de deux tritons, un sur chaque flanc : ils tiennent, l'un de la main droite, l'autre de la main gauche, une rame dont le manche repose sur la saignée du coude; l'un de la main gauche, l'autre de la main droite, une longue trompe dont ils soufflent. Au sommet de la proue, dans un évidement rectangulaire de la pierre, est un petit trou carré profond de $2 \mathrm{~cm}$. et destiné au scellement d'un objet de métal qui devait descendre au-dessous de ce trou, la pierre étant aplatie et noircie en cet endroit suivant une ligne verticale. A gauche et à droite de la coque se voient deux rames obliques en relief, beaucoup plus minces que celles des consoles précédentes. Sur la pierre, au nu du mur entre ces rames et la coque, on devine le profil de deux poissons en faible relief. Au-dessus des rames sont les préceintes et les bastingages. Le bord de gauche, vu de côté, montre nettement le premier croisillon du bastingage et au-dessus, à bord, un objet incliné de forme indéterminée ainsi qu'un casque vide vu de face avec son panache (fig. 8). De face, on voit à bord, de chaque côté, deux objets massifs qui ressemblent, surtout à gauche, à des cuirasses.

Le côté droit (fig. 9-10) comporte la préceinte et le bastingage à quatre croisillons. $\mathrm{Au}$-dessous, quatre rames inclinées vers l'arrière, figurées par des incisions rectilignes et, en relief, un gros poisson nageant à côté du navire et dans le sens de sa marche, la queue remontant en volutes pour s'épanouir à hauteur de la préceinte et du bastingage, débor- 
dant légèrement à son extrémité sur le méplat vertical qui limite à droite la sculpture. Au-dessus du bastingage on voit, de droite à gauche : deux paquets ficelés de lances ou de javelots inclinés vers l'arrière, - deux objets lisses et allongés (boucliers ? jambières ?) également inclinés vers l'arrière, dont le second se termine vers le haut par deux anneaux, - un bouclier rond vu de face, un casque vide vu de face avec son panache. Ld ou tant d'autres avaient vu des ballots de marchandises ou un mélange d'armes et de ballots ${ }^{1}$, il faut voir, avec Jullian et Espérandieu, uniquement des armes a bord de ce vaisseau.

b, à peine moins bien conservée que la précédente, n'avait pourtant jamais été regardée de près. Placée de la même façon, elle a, de face, sensiblement les mêmes dimensions : largeur, $1^{\mathrm{m}}, 20$; hauteur $0^{\mathrm{m}}, 92$; en queue, elle est moins longue : $1^{\mathrm{m}}, 72$, dont $0^{\mathrm{m}}, 98$ occupé par la sculpture (fig. 11). Sur la face, la partie sculptée a été diminuée en hauteur par un

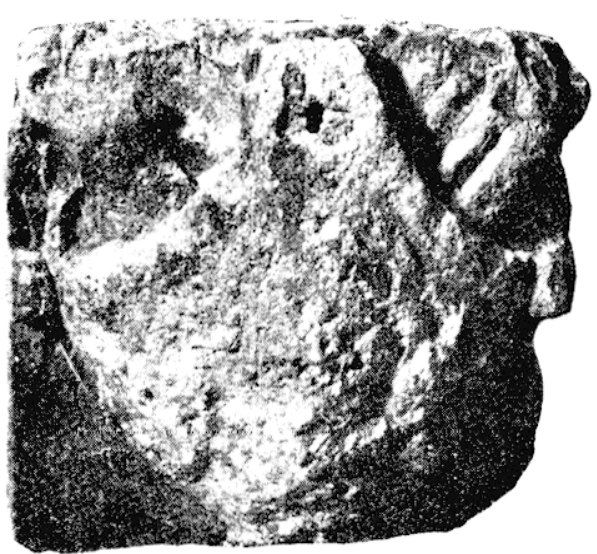

Fig. 6. - Face de la console a. évidement occupant toute la largeur destiné à recevoir la retombée de la voûte, ce qui réduit la hauteur visible et sculptée à $0^{\mathrm{m}}, 77$.

La proue est donc, de face, plus évásée que la précédente (fig. 12-13). Elle porte au sommet deux trous de scellement dont l'un contient au fond des restes de métal.

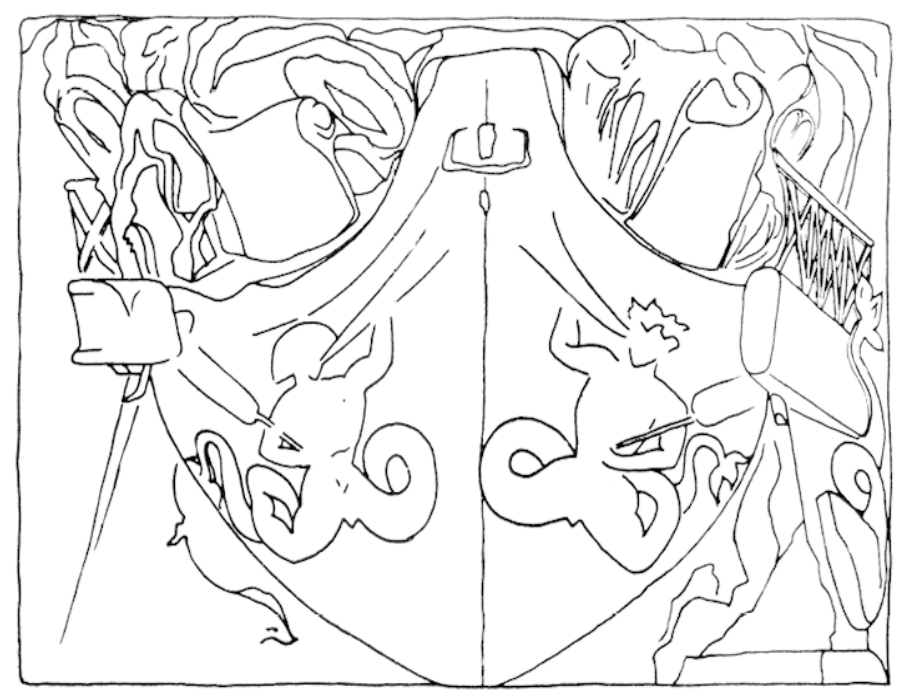

Fig. 7. - Face de la console a.
Les traces de deux tritons semblables à ceux de a se distinguent encore, surtout à droite, soit qu'il subsiste quelques frag. ments du relief, soit que l'arasement de celui-ci ait laissé sur la pierre les traces de son contour, traces qu'accuse la différence de couleur des parties arasées (blanc-gris) et du reste de la coque (jaune-rosé). A la différence de la coque a, celle-ci montre à sa base les trois ou quatre lattes inférieures de la carène, en léger relief. La moitié gauche de la sculpture

(1) Jollors, o. c., l. c.: « le vaisseau paraft chargé d'armes et de marchandises ». - Lenork, o. c., l. c.: "Ces navires sont chargés d'armes et de marchandises .. - Jullian, Rev. ét. anc., l. c.; Au seuil de notre Histoire, l. c. 
n'existe plus; à droite, on voit la rame oblique et mince en relief, et les restes de la préceinte et du bastingage. Entre la rame et la coque, la pierre au nu du mur est lisse. A bord, on ne distingue plus, à droite, qu'un objet massif qui a la forme d'une cuirasse. Le bord droit, vu de côté, montre la rame en relief, la préceinte, le bastingage avec l'amorce d'un croisillon, et, au-dessus du bastingage, les restes d'un objet indéterminé, ressemblant à celui qui occupe la même position en a.

Le long côté gauche se présente comme en a (fig. 14-15). Au-dessous de la préceinte et du bastingage dont on voit encore trois croisillons, sont les rames dirigées vers l'arrière. Mais, tandis qu'en a elles étaient au nombre de quatre et figurées en creux, ici on en compte neuf dont sept au moins sont en relief et deux peut-être (de chaque côté de la gueule du poisson) en creux : peut-être faut-il voir là la représentation maladroite de deux rangs de rames superposés. Le poisson est analogue au précédent. A bord, de

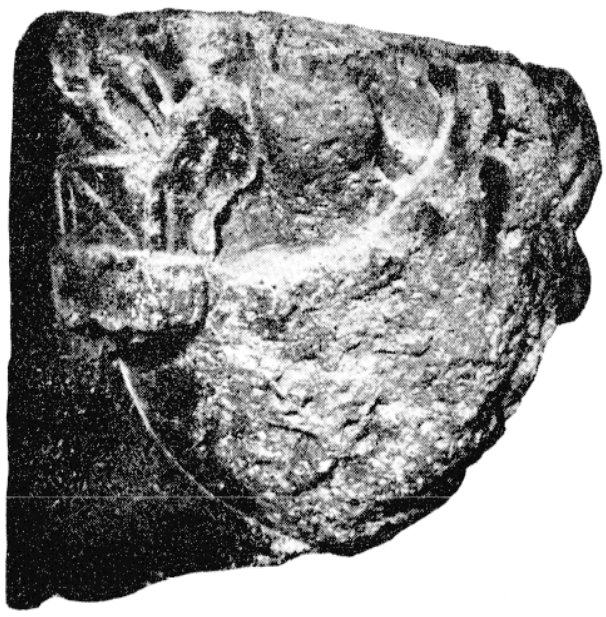

Fig. 8. - Côté gauche de la console a. gauche à droite, on voit : deux paquets ficelés de lances ou de javelots placés comme en a, puis un objet massif dont seule subsiste la partie inférieure, après quoi la sculpture n'existe plus.

Des proues de navires chargées d'armes : quelle sorte de navires? Et quelles armes?

Jullian l'avait déjà brièvement indiqué : malgré les armes, il ne s'agit point de galères mais de navires de charge ${ }^{1}$. Un navire de guerre ne se conçoit pas sans éperon ; c'en est l'élément essentiel, celui qu'on détache comme dépouille (soit sur les colonnes rostrales, soit sur les reliefs triomphaux : voir l'arc d'Orange), celui qu'on schématise jusqu'à la caricature sur les monnaies. Si l'on connait - quoiqu'on en ait dit — des voiliers et des barques de commerce à avant pointu ${ }^{2}$, on ne connaît pas de navires de guerre

(1) Rev. ét. anc., l. c.

(2) Sur de nombreux documents, les navires de commerce ou de pêche, barques ou voiliers, ont à l'avant une quille saillante et pointue, qui diffère de l'éperon des galères par l'absence de l'armature métallique, arme offensive toujours plaquée sur la quille en bois. - Voiliers: les exemples sont nombreux, notamment sur les monnaies (par ex. E. Muret, Cal. des monnaies gauloises de la B. N., nos 8611, 8613 (Atrebates); bronze de Commode, Diction. des Antiq., art. navis, fig. 5291, Cohen, III, 1883, n 635, p. 314 ; ct sur les mosaïques : citons le voilier narbonnais de la schola d'Ostie, Not. Scav., 1916, p. 327, fig. 5 et Grenier, Manuel d'arch. gallo-rom., VI ${ }^{2}$, p. 532 , fig. 170 ; le ponto de la mosaique d'Althiburus, Gauckler, Mon. Piot, XII, 1905, pl. IX-X et p. 142, fig. 22, et Greñer, o. c., p. 597, fig. 196 (pour d'autres navires de cette mosaïque, Gauckler place à tort la quille saillante à l'arrière où elle n'a que faire, et la publication doit être reprise intégralement) ; le voilier chargé d'amphores de Tébessa, GreniER, o. c., p. 604, fly. 201. Sur les bas-reliefs gallo-romains : le voilier au repos sur lequel deux hommes embarquent des ballots d'amphores (?), au Musée de Narbonne, Espérandieu, o. c., t. I, no 685. - Barques à rames : la barque de pêche d'une fresque étrusque de Corneto, La Roërie, Navires et Marins, De la 
sans rostre. Or, le profil et l'état de la pierre interdisent ici de supposer la présence primitive d'un éperon : les proues sont arrondies et ventrues. ce sont bien des proues de navires marchands. Ajoutons que les tritons qui ornent les flancs constituent un parti décoratif habituel aux navires de commerce, non aux galères : dans l'Antiquité comme de nos jours, les flancs des navires de guerre étaient nus ; ils portaient comme toute décoration, à l'avant, l'œil ou le poisson, tandis que les coques des voiliers de commerce étaient décorées de toutes sortes de sujets mythologiques.

Voiliers ou barques? Le caractère partiel de la représentation ne permet pas d'en décider; cependant la présence des rames - bien qu'il existe des voiliers pourvus de rames nombreuses, et des barques pourvues d'un mât de

rame à l'hélice, t. I, p. 55 ; les barques de pêche de la mosaïque de Sousse, Gauckler, Musée de Sousse, pl. VIII et pl. IX, 2. La barque de l'autel

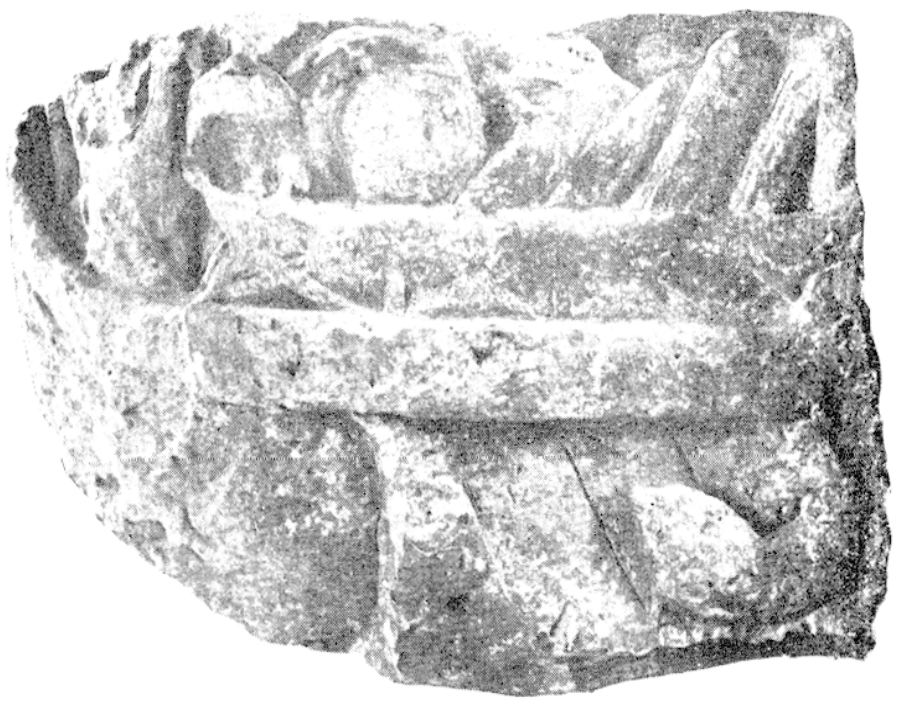

Fig. 9. - Long côté de la console a.

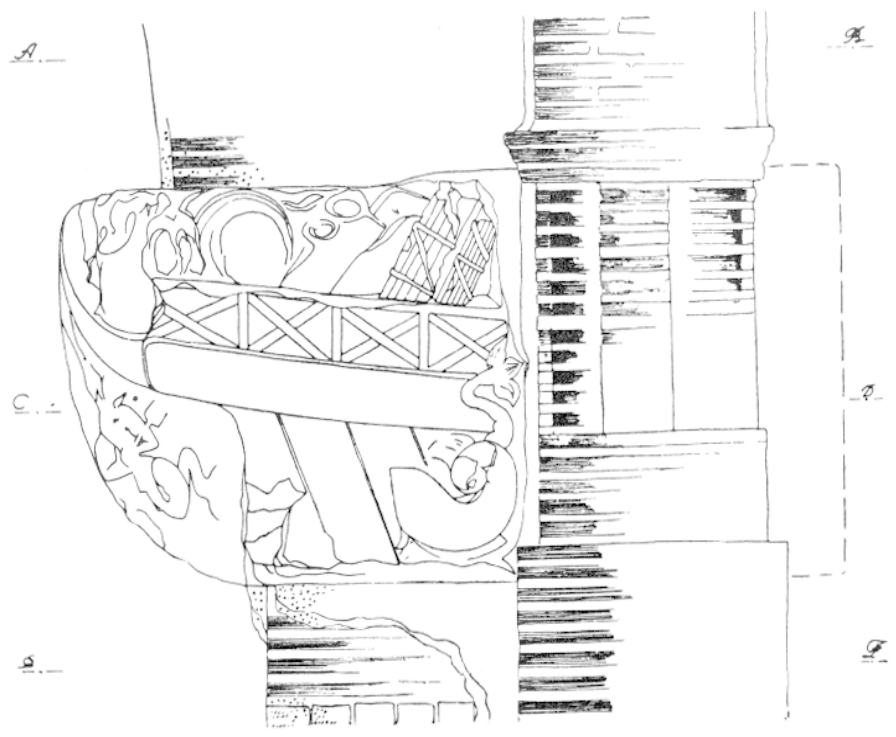

Fig. 10. - Long côté de la console a. de Marseille dite par erreur " arrivée de Lazare ", mal publiée par Espérandieu, o. c., t. I, no53, a en réalité une quille très saillante en forme de tête de dauphin à l'avant, Fernand Bexort, L'Autel de la place de Lenche à Marseille, Mém. Inst. Hist. Provence, XXI, 1946, p. 59-75, fig. p. 66; mais c'est peut-être un navire de guerre. 
secours - indique qu'il peut s'agir de barques. Mâtés ou non, ce sont des vaisseaux marchands à la coque relevée et arrondie aux deux bouts, et, puisque les rames sont figurées et non le mât, nous sommes en droit de songer plutôt à ces grosses barques fluviales dont on a, en Gaule, plusieurs représentations, et où le mât n'existe pas, ou n'est qu'accessoire ${ }^{1}$.

Quant aux armes, elles sont figurées sans proportions réelles (le casque, par exemple, a la

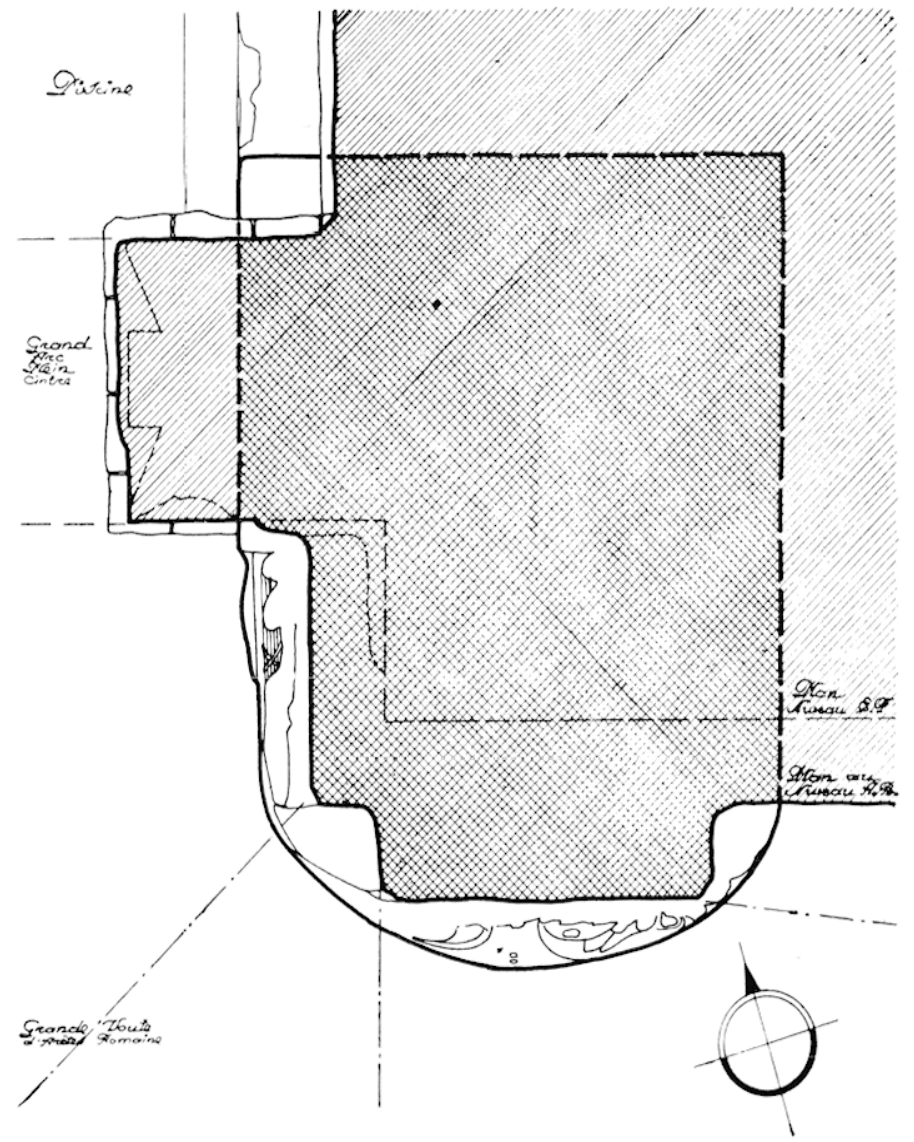

Fig. 11. - Plan de la console b. même dimension que le bouclier, fig. 9-10), comme sur une frise, le sculpteur ayant tiré parti de la bande restée libre entre le bastingage ou la proue et la limite supérieure de la pierre. Elles se présentent de façon décorative, comme sur les bandeaux des mausolées ou des arcs gallo-romains, dont on peut voir tant de morceaux, par exemple, à Arles ou à Narbonne. Cie sont des armes romaines courantes, toutes défensives sauf les javelots. On n'y peut reconnaitre aucun caractère gaulois, et il est inutile de s'arrêter à l'indication d'Espéran-

(1) La forme du navire de commerce, mâté ou non, est en effet, couramment relevée aux deux bouts. Voiliers : c'est la forme de la corbita de la mosaïque d'Althiburus, Gauckrer, o.c., p. 132, fig. 8 et Grenier, o. c., p. 588, fig. 190. Monnaies gauloises : E. Muret, $o . c$. nos 8743, 8744 (de Latour, Atlas de monnaies gauloises, pl. XXXV, Ménapes), 9445 (ibid., pl. XXXIX, Bolens). Bas-reliefs : à Narbonne, Esperandiev, o. $c$., n ${ }^{08} 678,686,687,690$. - Barques : barques hâlées du soubassement du mausolée d'Igel, ibid., n 5268, et du bas-relief de Cabrières d'Aigues (Musée d'Avignon), ibid., $\mathrm{n}^{\circ} 6699$; barque de la stèle de Blussus à Mayence, ibid., $\mathrm{n}^{\circ} 5815$; barque inédite de l'autel de Glaukias au Musée Borély (ibid., n 80 ) ; barque dite d'Ulysse du bas-relief de Carlsruhe, Espérandiev, Recueil... de la Germanie, no 344 . 
dieu et de Jullian supposant, à bord de ces navires, des trompettes gauloises ${ }^{1}$.

$\mathrm{Ni}$ la forme du navire, ni celle des armes ne peuvent donner une indication chronologique dans les limites de l'Empire romain. Mais le style des sculptures, large et puissant, non sans délicatesse dans le modelé des tritons et des casques, ne saurait dater du Bas-Empire. D'ailleurs, le mode de construction de l'édifice auquel elles appartiennent, où la proportion de la brique est faible par rapport à celle du petit appareil de moellons, et où les portes des sous-sols sont construites de façon puissante en pierres de taille à bossages, est certainement antérieur au Bas-Empire : aussi bien,

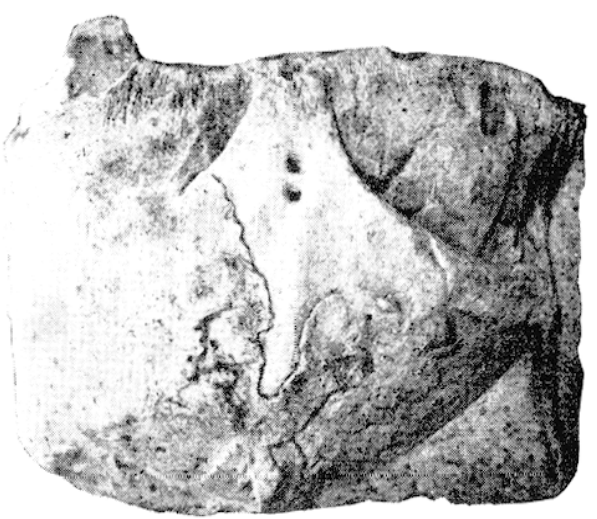

Fig. 12. - Face de la console b. on admet aujourd'hui avec de Pachtère que la ville de la rive gauche était détruite à la fin du III ${ }^{\mathrm{e}}$ siècle $^{2}$. Le monument et ses sculptures sont probable-

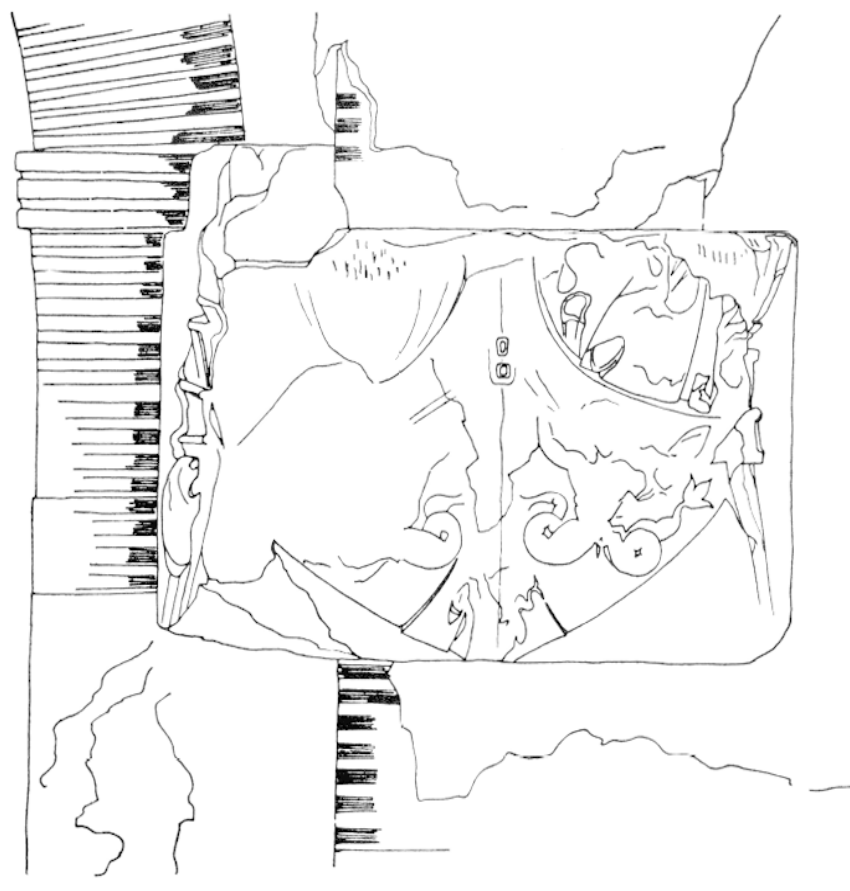

Fig. 13. - Face de la console b. ment du III ${ }^{\mathrm{e}}$ siècle, peutêtre du $I^{e}$.

L'interprétation de ces barques chargées d'armes est d'autant plus ardue que des consoles de cette forme ne se trouvent nulle part ailleurs dans l'architecture antique. Le fait est curieux, car la forme du navire convient particulièrement bien à cette fonction : un ovale

(1) Espérandieu, no $3204 . \mathrm{De}$ même Jullian, Rev. ét. anc., l.c., qui parle, en outre, de haches, et distingue un faisceau de lances et un faisceau de javelots, en ajoutant d'ailleurs : con peut hésiter sur chacune de ces attributions $"$ : c'est dire trop, et trop peu.

(2) De Pachtère, o. c., p. xxiv, 117, 170. Des gradins des Arènes ont été remployés dans les fondations de l'enceinte de la Cité au Bas-Empire, ibid., p. 80, 142, 146, n. 2. 
tronqué par le haut et pointu à la base est, au point de vue architectonique comme au point de vue esthétique, tout à fait adéquat au soutien d'une retombée de voûte; ce sera la forme ordinaire des culots supportant la base des ogives dans l'architecture médiévale. Mais l'explication est insuffisante. On pourrait aussi penser que les armes ne sont qu'un poncif et un bouche-trou : l'artiste ('hargé de représenter un navire aurait, pour "faire beau " et aussi pour utiliser toute la surface à sculpter, ajouté la panoplie en s'inspirant des cartons faits pour les monuments publics décorés - en ces siècles de fer, les armes régnaient dans l'art comme dans la vie. Cie sont là explications paresseuses : il en est pour tout problème.

Si, les rejetant, nous cherchons au sujet choisi un caractère symbolique, plusieurs possibilités se présentent :

10 Symbole cosmique : ces sujets nauti-

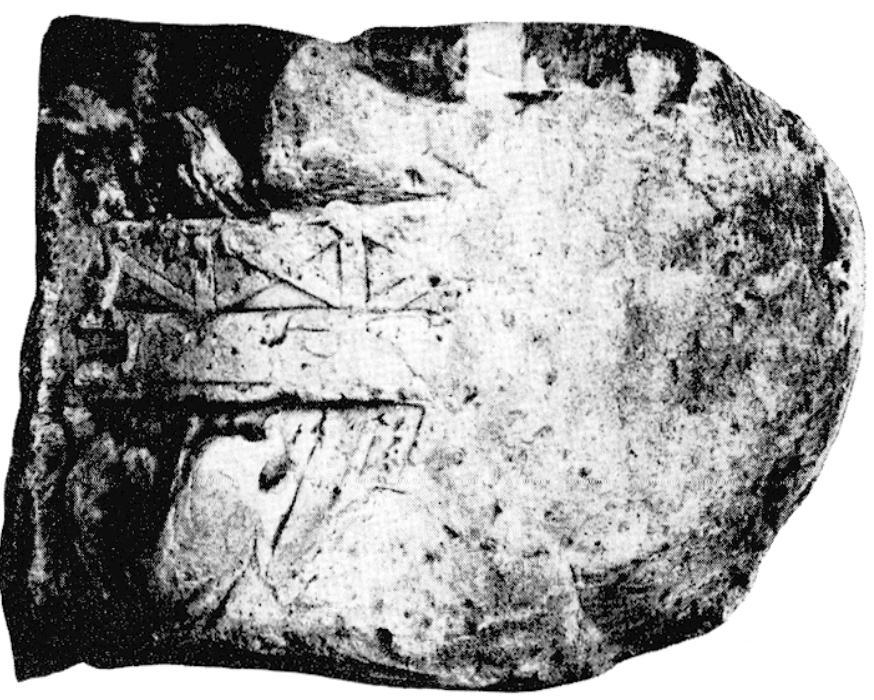

Fig. 14. - Long côté de la console $b$.

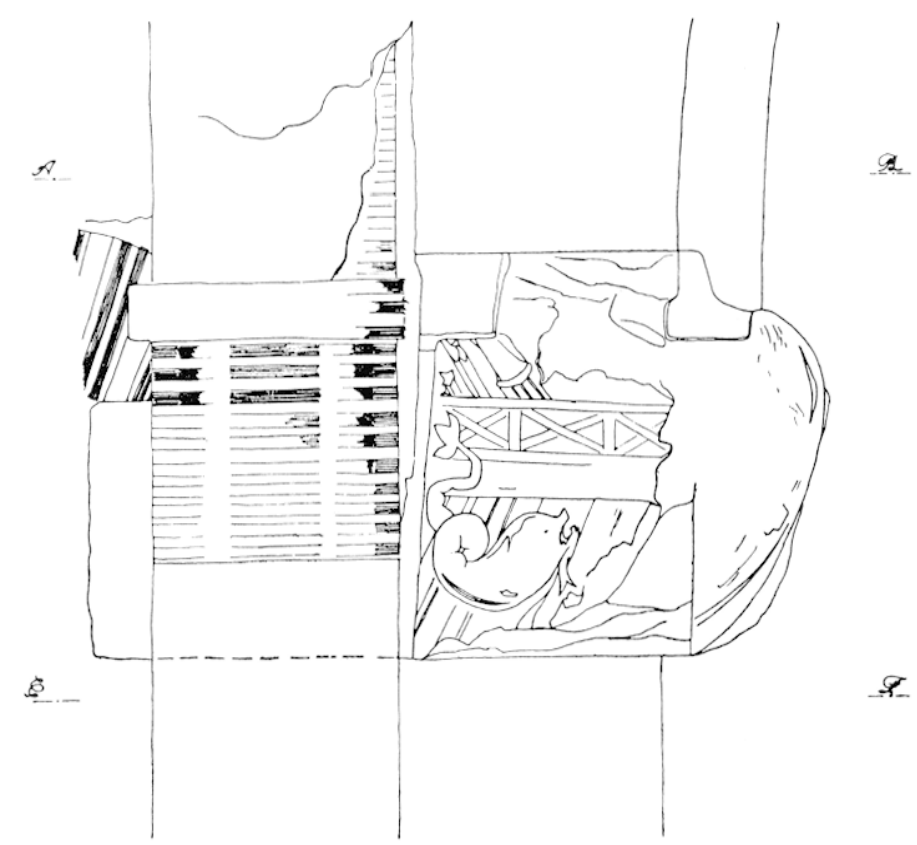

Fig. 15. - Long côté de la console b. ques feraient partie d'une décoration dont les autres parties seraient également, dans d'autres salles, des symboles des éléments, - la terre, l'air, le feu. Les armes seraient 
un signe de domination sur l'empire des ondes. - Cette hypothèse séduisante nous paraîtra fragile jusqu'à nouvel ordre : on ne connaît pas de décoration d'ensemble à l'échelle du monde dans un édifice romain; une telle décoration conférerait au monument un caractère impérial que rien n'autorise, et conviendrait mal à une salle de bains ;

$2^{\circ}$ Symbole thermal : décoration de nature aquatique. - Soit : mais les armes, alors, n'ont plus aucun sens ;

30 Symbole triomphal : commémoration par une série de trophées d'une victoire navale. - Cette hypothèse, à laquelle Jullian avait songé sans s'y arrêter ${ }^{1}$, est, tout d'abord, sans soutien historique : on cherche en vain la victoire navale qui, au II $^{\mathrm{e}}$ ou au III $^{\mathrm{e}}$ siècle, aurait pu être commémorée de façon si éclatante à I utèce. De plus, il serait étrange qu'on n'eût pas choisi le type des navires de guerre pour un trophée; on peut répondre que, sur les grands fleuves de la Gaule, les barques fluviales ont fini par être incorporées aux flottilles militaires ; le fait s'est certainement produit au Bas-Empire, il aurait pu se produire auparavant. Mais il est une objection plus forte : un trophée est un ensemble sculptural qui réclame une place d'honneur. Ni la fonction utilitaire de consoles, ni leur situation à plus de $7 \mathrm{~m}$. de haut, ni surtout le cadre d'une salle de bains, ne lui conviennent ${ }^{2}$;

$4^{\circ}$ Emblème militaire administratif : la salle de bains serait annexée à la résidence officielle du chef d'une flotte fluviale ; ou encore, la présence à Lutèce de ce personnage important aurait pu donner assez de lustre à la ville pour qu'on la rappelât dans la décoration d'un édifice public. - Rien de tel n'est attesté à Lutèce avant la fin du rve siècle au plus tôt. C'est seulement à cette époque que Paris est le lieu de résidence du préfet de la flottille de la Seine, la Classis Anderetianorum $^{3}$. Il ne semble pas qu'on soit en droit de postuler l'existence au $\mathrm{III}^{\mathrm{e}}$ ou au $\mathrm{rI}^{\mathrm{e}}$ siècle d'une telle flottille (qui est une institution typique du BasEmpire, destinée à défendre le cours des fleuves et leurs points de passage prin-

(1) Rev. ét. anc., l. c.

(2) Cette fonction, ce cadre seraient même indécents. Les représentations commémoratives de victoires navales sont toujours en bonne place : la colonne rostrale de Duilius (Musée du Capitole) s'élevait sur le Forum; les dépouilles navales figurent en quatre panneaux sur l'arc d'Orange, deux sur chacune des faces principales; le trophée naval de Saint-Bertrand-de-Comminges, constitué d'un avant à éperon avec figure de proue féminine, s'élevait au centre d'un groupe sculpté, cf. G.-Ch. PicArD, Trophées d'Auguste à St.-B. de C., Mém. Soc. Arch. du Midi de la Fr., XXI, 1947. Les statues de Victoires sur base en forme de proues occupaient elles aussi, par nature, des places d'honneur : sur ces bases, cl. l'étude de J. Marcadé, Sur une base lindienne en forme de proue, Rev. arch., 1946, p. 147-152, qui se demande si le goût pour les proues décoratives ne remonterait pas au bûcher élevé par Alexandre pour Héphaistion à Babylone, et que nous connaissons par Diodore de Sicile, XVII, 115, 2 : autour du soubassement, 240 proues, or sur rouge, surmontées de statues d'archers et d'hoplites.

(3) Not. Dign., Occ., XLII, 23. 
cipaux contre les Barbares), puisque la première vague vraiment puissante des invasions qui a dû détruire le quartier de la rive gauche de Lutèce date de la fin $d u$ III $^{e}$ siècle. Le "Palais des Thermes " est un monument de l'époque de la prospérité, non des troubles et de la décadence. Il faudrait, pour pouvoir l'attribuer à l'époque de la création de la flottille, faire remonter celle-ci au moins jusqu'au début du règne de Dioclétien, et admettre que le monument, dont le mode de construction ne saurait être postérieur au II $^{\mathrm{e}}$ siècle, a été détruit presque au lendemain de sa construction : double postulat;

$5^{\circ}$ Emblème professionnel : le monument est lié à l'activité d'une corporation ayant trait au commerce fluvial et, plus précisément, au transport des armes. - Deux réalités historiques sont ici en jeu : la corporation des Nautes Parisiens, et les fabriques d'armes du Nord et de l'Est de la Gaule.

Les nautes fixés à Lutèce étaient assez riches pour y faire construire un édifice de l'importance du "Palais des Thermes ". Jullian, sous l'influence du livre de de Pachtère qui rejetait l'hypothèse des thermes, a songé tout d'abord à une schola ${ }^{1}$. Mais cette identification ne se soutient que si la salle centrale, décorée aux emblèmes de la navigation, peut être la salle principale d'une schola: or, la présence de la piscine oblige à y voir une salle de bains publics, les dimensions dépassant de beaucoup celles d'un bain privé, voire corporatif. Aussi Jullian, revenant sur son opinion, se rangea-t-il plus tard à l'hypothèse des thermes ${ }^{2}$, mais sans abandonner l'idée d'un lien avec la corporation des nautes ${ }^{3}$. C'est ce lien que notre meilleure connaissance des consoles doit nous permettre de préciser.

Faut-il s'en tenir à l'idée de Jullian, émise à une époque où il mûrissait sa première hypothèse d'une schola: les nautes auraient, dans cet édifice, perpétué le souvenir de leur commerce principal, dont l'objet aurait été les armes fabriquées en Gaule ? Dans l'état actuel de nos connaissances, cette hypothèse

(1) Hist. de la Gaule, IV (1914), p. 393, n. 5 : a Il n'est pas impossible que l'édiflce... ait servi de schola ou maison corporative aux Naulae Parisiaci. Ce qui me l'a fait supposer, c'est la présence, dans ce qui est évidemment la plus grande salle de l'édifice, de consoles sculptées figurant des avants de navires chargés de faisceaux d'armes, cuirasses, casques, etc.; et ce pourrait être l'emblème des nautes. Car les corporations possédaient leurs emblèmes, leurs armes parlantes (Rostowtzew, Bleitesserae, p. 96-97) : c'est ainsi que les utriculaires de Cavaillon $(C . I . L$., XII, 136) représentaient une outre sur leurs jetons ou tessères (BABELon et BLANCHET, Bronzes, $\mathrm{n}^{\mathrm{n}}$ 2315) et que les scapharii d'Espagne avaient un navire de charge entre autres emblèmes (C. I. L., II, 1168-1169) *.

(2) $0 . c$. , VI $(1920)$, p. 415, n. 1 : a Je dois avouer que les dimensions et les dispositions architecturales et le grand conduit souterrain me paraissent convenir plus à des thermes qu'à tout autre type de monument connu ». - Dans Le Paris des Romains (1924), ce sont des thermes qu'il décrira.

(3) C'est le dernier état de sa pensée sur cette question: Au seuil de notre Histoire, I (1930), p. 243, n. 1, où il corrige l'opinion professée en 1905-1906 au Collège de France (11 le leçon du cours).

(4) Rev. ét. anc., XVI (1914), p. 216 : "cet avant de navire ne serait-il pas l'image des bateaux de charge qui, pour le compte de l'État, transportaient les armes fabriquées pour l'armée par les établissements libres ou publics? Et alors, pourquoi ne point penser ici aux nautes de Paris ? ^. - Aux fabriques d'armes pense, lui aussi, M. A. Piganiol, Comptes rendus Acad. Inscript., 1946, p. 208. 
pourrait se formuler ainsi : à un moment que nous ignorons, avant le Bas-Empire, les nautes parisiens ont passé avec l'État un contrat leur assurant le transport des armes ; ce contrat leur valant un surcroît de bénéfices, ils ont alors fait construire à Lutèce; tout près de la Seine, des thermes publics dont quelques salles étaient peut-être réservées à leurs bureaux, et ont rappelé dans la décoration du frigidarium central le caractère de leur activité nouvelle. 11 reste que : des fabriques d'armes relativement proches du réseau fluvial contrôlé par les nautes parisiens ne sont pas attestées avant la fin $d u$ Ive $^{e}$ siècle au plus tôt ${ }^{1}$, qu'il est douteux que les nautes aient travaillé pour l'État au III ${ }^{\mathrm{e}}$ siècle, et encore plus incertain que le transport des armes ait été l'objet principal de leur activité.

Aussi croyons-nous d'une autre nature le lien qui unit le "Palais des Thermes " aux nautes parisiens. Dans ces barques fluviales et dans ces armes, nous verrions volontiers une composition flatteuse pour la vanité des riches armateurs qui, socialement parlant, étaient certainement les "rois du pays". Ils étaient assez puissants pour afficher leur générosité sous forme d'emblèmes dans un édifice que leur prospérité pouvait offrir à la cité. Il entrait dans leurs usages de se faire représenter armés de pied en cap, comme on les voit sur l'autel dédié au ${ }^{\text {er }}$ siècle à Tibère et à Jupiter trouvé dans la Cité en 1711 : et ce costume avantageux exprimait d'ailleurs une réalité, car, bien qu'ils fussent une force civile, les nautes assuraient sans doute eux-mêmes la protection de leurs convois ${ }^{2}$. Dans ces conditions, il parait naturel qu'ils aient volontiers rappelé dans leur emblème ce rôle para-militaire qui comportait une part de dévouement civique.

De toutes celles que nous avons examinées, la dernière hypothèse nous parait la plus chargée de réalité. Les fouilles en cours, si elles nous livrent son plan complet, préciseront peut-être la destination du "Palais des Thermes ": nous savons déjà que la salle décorée de consoles est un frigidarium de thermes publics. Que ces thermes publics soient indépendants ou annexés à des locaux

(1) Jullian cite bien une fabrique d'armes chez les Éduens sous les Sévères, à Brève, Rev. ét. anc., l. c., p. 216, n. 2, d'après $C$. I. L., 2828, et indique que le monument de Cluny lui parait c contemporain ou voisin des Sévères *. Mais cela sumt-il à soutenir une hypothèse aussi précise ? Les autres fabriques d'armes nous sont connues seulement par la Notitia Dignitatum, en plein Bas-Empire : Occ. IX, 35, Suessonensis...; 36, Remensis spatharia; 39, Ambianensis spatharia et scutaria.

(2) J. Toutain, Le monument des Nautes Parisiens et le commerce fuvial sur la Seine au Ier siecle de l'ére chrétienne, Mém. Soc. du *Vieil Argenteuil ", Bull. no 13, 1941, 7 p., approuvé par R. LANTizR, Rev. arch., 1945, p. 159-160 et par A. Grenier, Rev. ét. anc., 1942, p. 130. Tandis que Jullian voyait dans la tenue militaire des nautes un costume religieux archaïque (avec DE PACHTĖRE, Rev. ét. anc., 1907 (p. 263 ss.) ou l'indice de quelque rôle officiel (ibid.; 1914, p. 216, n. 3), M. Toutain estime que les bateliers parisiens étant responsables de leur chargement envers leurs clients, et la Gaule indépendante ni la Gaule romaine n'ayant connu de police d'État, "il est tout naturel qu'ils fussent armés pour se defendre contre toute tentative de vol . 
corporatifs ou autres, ils s'élèvent dans la ville des nautes, et tout près du fleuve sillonné par leurs barques. Cet argument local se renforce du fait que l'édifice est le seul monument antique connu décoré de consoles en proues de navires, et qui plus est : de navires marchands. Quelle que soit la nature du lien qui pouvait unir les nautes au "Palais des Thermes", ce lien doit donc être maintenu. Aussi bien, il s'impose invinciblement à l'esprit dans la salle du Musée de Cluny, où les proues de navires semblent veiller sur l'autel des nautes en armes.

$$
\text { ** }
$$

Le sol de Paris a livré plusieurs sculptures ornées de sujets "aquatiques", vestiges de monuments dont ils attestent l'existence et la date approximative.

Le morceau le plus médiocre (Espérandieu, $n^{0} 3182$ ) est un bloc de frise en pierre qui, après avoir appartenu au Musée Carnavalet, est aujourd'hui égaré (ancien inventaire de la collection lapidaire, no 195). Trouvé en 1906 au Marché aux Fleurs (fouilles du Métropolitain), où passait l'enceinte du Bas-Empire, dans laquelle il était probablement remployé, il avait dû appartenir à un monument situé en un autre point de la ville. - Un lion marin se dirige vers la droite. Le style raide, le relief plat, la sculpture peu fouillée, indiquent une époque tardive : seconde moitié du $\mathrm{II}^{\mathrm{e}}$ siècle, au plus tôt.

Un autre bloc de pierre (Espérandieu, $\mathrm{n}^{\circ} 3160$ ), au Musée Carnavalet (anc. invent. $\mathrm{n}^{\circ}$ 101, nouvel invent. $\mathrm{n}^{\circ} \mathrm{AP} 69$ ), provient des fouilles pratiquées à la Caserne de la Cité au siècle dernier. - Sculpté en fort relief, un personnage barbu, nu, est assis sur la queue recourbée d'un monstre marin dont l'avant a disparu; la queue d'un autre monstre s'amorce à gauche. Le style est puissanl, mais sans finesse; la sculpture a du mouvement; elle évoque les productions du $\mathrm{II}^{\mathrm{e}}$ siècle.

Publié par Espérandieu ( $\left.n^{\circ} 3162\right)$ sans provenance, un petit fragment de frise en pierre de Saint-Leu au Musée Carnavalet (anc. invent. no 81, nouvel invent. no AP 6) a été trouvé au cimetière Saint-Marcel, remployé comme couvercle d'un sarcophage d'enfant des Ive-ve siècles. Une femme nue dont la main droite semble tenir l'extrémité d'un voile est assise sur un cheval marin nageant parmi des plantes. Sur le bord inférieur, fait pour être vu d'en bas, s'étale un décor floral. A droite, un arbre sert de cadre à la scène. Le style est élégant, le mouvement est bon, et la silhouette de la Néréide bien campée. Le relief, assez sec, ne manque pas de vigueur : ces qualités permettent d'attribuer l'œuvre au II $^{\mathrm{e}}$ siècle; elle ne serait pas déplacée à la fin du $\mathrm{I}^{\mathrm{er}}$.

Des bas-reliefs de ce genre sont courants dans tout le monde romain, et notamment en Gaule où ils ornaient des monuments de destinations différentes: arcs de triomphe (Orange, Besançon : Espérandieu nos 260, 5270), théâtres (Lillebonne : ibid., nos 3089,3126 , Champlieu : no 3822), temples (Champlieu : $\mathrm{n}^{\text {os }} 3814,3818,3821,3823,3826-2832,3838$ ), thermes (Sens : no 2856, fig. 14 et 16), sarcophages (Athis-Mons: $n^{0} 7175$ ), etc... Et l'on en trouve dans bien des villes qui n'ont point de rapport avec la navigation. - Les deux sculptures de 
Paris que nous attribuons au $\mathrm{II}^{\mathrm{e}}$ siècle ont-elles appartenu au "Palais des Thermes »? La chose n'est pas impossible pour le $n^{0} 3162$, bien que le style paraisse plus ancien, en tout cas plus raffiné que celui des consoles. Pour le $n^{\circ} 3160$, la provenance semble interdire cette attribution. Quant au n ${ }^{\circ} 3182$, dont la provenance réelle est inconnue, son style est nettement inférieur à celui des consoles. On ne peut donc rattacher à coup sûr aucun de ces documents au "Palais des Thermes ".

Encore moins, à la navigation parisienne. Par contre, à cette activité peuvent se rapporter deux autres pièces du Musée Carnavalet.

La première est une base de colonne en pierre trouvée en 1848 dans la cour de la Sainte-Chapelle, près du quai des Orfèvres (anc. invent. $\mathrm{n}^{0} 45$, nouvel invent. $\mathrm{n}^{\circ}$ AT 85 ). Cette base à deux tores, petite (diam. $0^{\mathrm{m}}, 50$, haut. $0^{\mathrm{m}}, 38$ ), est creusée, sur un seul côté, d'un sillon oblique profond qui doit être dû au frottement d'amarres, cordes ou câbles' ${ }^{1}$. Cette base a pu appartenir à une installation portuairc dc l'île.

La seconde pièce est une petite proue de navire d'une singulière qualité, trouvée vers 1862 dans l'ancienne cour des Comptes, au sud-ouest de la Sainte-Chapelle. Egarée depuis, retrouvée par $M^{\text {me }}$ Durand-Lefebvre en 1941, elle est aujourd'hui le joyau de la collection lapidaire du Musée. Sa description par $\operatorname{Read}^{2}$ a été reproduite par Espérandieu ( $\mathrm{n}^{\circ} 3161$ ). $\mathrm{M}^{\mathrm{me}}$ Durand-Lefebvre, qui publiera une étude approfondie de la pierre dans le Bulletin de la Société Nationale des Antiquaires de France, a bien voulu nous autoriser à faire état de ses conclusions, auxquelles, après examen du document, nous souscrivons entièrement.

La proue est taillée dans une pierre blanche ressemblant à du marbre à s'y méprendre, et dont on trouve l'analogue à Bourré (Oise). Il s'agit d'un bloc architectonique (haut. $0^{\mathrm{m}}, 22$, long. $0^{\mathrm{m}}, 32$, larg. $0^{\mathrm{m}}, 25$ ) dont la queue est cassée, mais qui était fait pour être engagé dans une paroi, car l'objet ne peut, reposer sur la quille aiguë du navire. Au sommet, un trou de scellement carré nous indique que la proue supportait un autre élément, colonnette, petit pilastre ou statuette. La proue est trop petite pour supporter la retombée d'une voûte : ce n'est donc point une console. C'est plutôt un ornement, fixé au mur d'un monument dont la nature est impossible à définir, et qui se trouvait sur l'emplacement du plus ancien palais de l'ile, dont il faisait peut-être partie.

A la base de la proue, qui est assez peu ventrue, une cassure ovale dont les bords constituent un départ de relief nous invitent à restituer un éperon. Une mince préceinte double est surmontée d'un bastingage à croisillons de chaque côté. Les deux flancs sont décorés de sujets identiques, qui ne sont pas inhérents au navire, mais représentent une scène indépendante : sur les flots ondulés vogue un centaure de mer (torse humain, train de cheval avant, queue recourbée de monstre marin, tête barbue à longs cheveux, portant des cornes, peut-être des pinces de homard), le bras droit levé main ouverte, la main gauche tenant un objet plat et rond, probablement une patère. Sur sa croupe est assise une femme, de dos, dont la main gauche levée au-dessus de la tête tient un voile qui retombe le long de son côté gauche et, laissant le dos découvert, passe sous elle

(1) Papiers Vacquer, t. XLIV, fol. $171 \mathrm{v}^{\circ}$, à la Biblioth. des Trav. hist. de la Ville de Paris. Albert Lenoir, Mem. Soc. Antiq. de France, 1846, p. 331. Voir p. 142, addenda.

(2) Bull. de la Soc. nat. des Antiq. de Fr., 1869, p. 71. 
pour venir recouvrir les genoux. Le couple est classique : une Neréide, ou Amphitrite, et Océan. Il est précédé d'un Amour qui tient de sa main gauche l'umbella et, les jambes élégamment croisées, la tête tournée vers Océan, guide sa marche : on le retrouve en tête du cortège d'Amphitrite sur des documents du ier siècle. Il ne vole point, parachutiste avant la lettre, à l'aide de l'umbella : il la porte solennellement, comme les petits pages porte-parasols si chers à l'Orient'.

D'après les caractères de la sculpture (absence de trépan, excellente qualité du style, coiffure de la Néréide), $M^{\text {me }}$ Durand-Lefebvre propose avec raison de dater cette pièce de choix de la fin du I $^{\text {er }}$ siècle ou du début du II $^{\mathrm{e}}$. Nul doute que, au contraire des reliefs précédents, cette proue de navire, unique, elle aussi, en son genre, ait, comme les consoles du "Palais des Thermes ", quelque rapport avec la navigation parisienne ${ }^{2}$.

Dûment attestée à l'époque romaine, l'activité de la navigation fluviale dans la région de Paris était certainement antérieure à la Conquête : on sait que pendant la campagne des Gaules, Labiénus put s'emparer d'une flottille gauloise importante groupée sur la Marne et la Seine entre Meaux et Melun, et notamment à Lutèce ${ }^{3}$. D'autre part, si les nautes étaient organisés sous Tibère

(1) Pour les comparaisons, nous renvoyons à l'étude de $\mathrm{M}^{\mathrm{m}}$ • Durand-Lefebvre : notamment, on trouve l'Amour à l'umbella sur les plaques de terre cuite de la Porta Pia à Rome (cortège d'Amphitrite), H. von Rohden, Die antiken Terracolten, p. 29 ; G. Pietro Campana, Antiche opere in plastica, pl. X, p. 52. - Signalons que, sans l'umbella, il soutient le voile de la déesse portée par un Océan-triton barbu sur un sarcophage trouvé à Athis-Mons (Seine-et-Oise), Espérandieu, no 7175. Le centaure de mer est représenté sur le monument du port à Milet (époque d'Auguste), Milet, I, 6, 1922, p. 61, flg. 73 ; p. 70, fig. 86. Le couple est fréquent sur les mosaïques, notamment en Tunisie, et à Antioche (Antiochon-the-Oronles, II, The excavations 1933-1936, Princeton, 1938, pl. 23, n० $33 \mathrm{c}$ ).

(2) On peut imaginer un monument du type du Monument du Port à Milet, dont le soubassement était flanque de deux proues de navires de guerre, Milel, I, 6, 1922, p. 63, fig. 76-78; p. 70, fig. 86. Les proues de navires n'ont pas disparu de la décoration parisienne, car leur multiplication a été favorisée par l'adoption de la nef des " marchands de l'eau " comme emblème de la cité : c'est ainsi que les réverbères principaux de la place de la Concorde sont des colonnes rostrales dont les proues portent dans un médaillon l'effigie de la nê médiévale; ceux de la place de l'Opéra sont du même type, mais d'un modèle un peu différent, sans la nef. On ne saurait, à l'heure actuelle, établir historiquement une fliation directe entre les "marchands de l'eau ", dont nous connaissons l'emblème seulement depuis le xII $^{\circ}$ siècle, et les nautes antiques. Ils se confondent pourtant dans la tradition, car on ne peut attribuer qu'à la proximité de la voie fluviale, artère vitale de Paris, les proues sculptées qui ornent les guichets du Louvre face au Pont Royal, et celles qui flanquent la base des quatre pilastres surmontés de chevaux ailés du Pont Alexandre III : à raison de trois par pilastre, ce sont des proues de navires marchands chargées de fruits de terre et de mer, de fleurs et de feuillages, ornées de figures humaines; l'une d'elles (pilastre sud-ouest) est chargée d'armes antiques : glaives, boucliers, haches, posées pêle-mêle avec une enseigne portant les lettres RF ! S'agit-il d'une imitation des proues de Cluny? ou simplement d'une composition pseudo-antique pour laquelle on peut admettre l'hypothèse du c poncil , (armes sur un bateau marchand) que nous avons écartée à propos des sculptures antiques?

(3) CÉs., De b. gall., V, 5, 2 ; VII, 58, 4 ; VII, 60, 4. 
comme l'atteste la dédicace de l'autel de la Cité, leur corporation devait avoir déjà un longr passé derrière elle, et C. Jullian et de Pachtère ont insisté avec raison sur l'aspect archaïque de leur costume ${ }^{1}$.

De cette existence de la navigation parisienne aux temps de l'indépendance, nous croyons trouver la première preuve matérielle sur une monnaie d'or des Parisii découverte au Pont Louis-Philippe et dont l'avers porte une tête d'homme sous laquelle est figuré un objet qui n'a jamais été défini ${ }^{2}$ (fig. 16). Get objet allongé dans le sens horizontal, renflé et dont l'extrémité recourbee fait pendant à une mèche de la chevelure, nous parait trop important pour être un simple ornement décoratif : le schématisme est en effet ici peu poussé, et

l'objet garde une forme
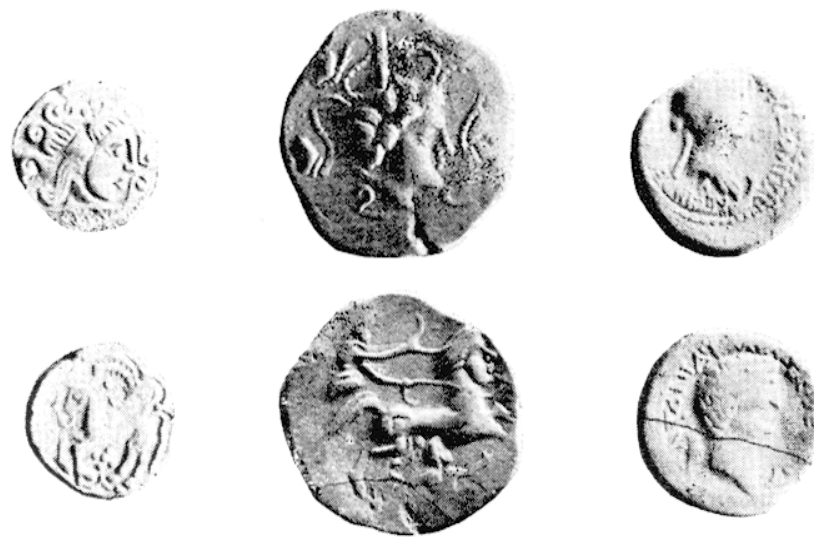

Fig. 16. - Monnaies des Parisii à gauche, des Namnètes au milieu, de Cléopâtre à droite. nettement caractérisée. Cette forme n'est point celle d'un poignard, ni d'une épée; d'un joug, non plus ; d'un collier, moins encore. Sa volute et sa courbe inférieure interdisent d'y voir un socle ou un monument supportant la tête. Par contre, elle convient parfaitement à une barque aux extrémités relevées, dont une seule est ici complètement visible.

Notre opinion est confirmée par l'existence de navires en même place sur d'autres monnaies. Tout d'abord, des monnaies gauloises, frappées par d'autres peuples navigateurs, les Namnètes de l'Océan ou les Andécaves de la basse Loire : sur l'une d'elles, on voit, sous la tête d'Ogmios, un objet où Hucher a reconnu le profil d'un navire aux extrémités anguleuses, orné d'une figure de proue animale (fig. 16) ${ }^{3}$. En second lieu, une monnaie d'Antoine et Cléopâtre

(1) Rev. ét. anc., 1907, p. 263 ss.

(2) E. Muret, Calal. des monnaies gauloises de la B. N., no 7796 (1 gr. 67) ; voir aussi le no 7798 ; toutes deux dessinées dans de Latoun, Atlas de monnaies gauloises, pl. XXXI.

(3) E. Hucher, L'art gaulois, ou les Gaulois d'après leurs médailles, I, 1868, p. 11, pl. 35, 1 : pièce de la collection Hucher, où la figure de proue est parfaitement visible; autre exemplaire dans MURET, o. c., n' $6728=$ de Latour, o. c., pl. XXI, où la figure de proue, comme sur notre fig. 16 b, est effacée; HUCHER, o.c., II, 1873, p. 93, fig. 148 (autre exemplaire). Nous ne suivons pas Hucher quand il voit dans ce navire un symbole solaire ou funéraire, ni quand il interprète sur d'autres monnaies un objet en forme de joug ou de mors comme une dégénérescence du navire (II, fig. 149, 150, p. 95), ni quand il reconnait un navire sur d'autres monnaies des Namnètes (I, pl. 93, 2). 
nous apprend que l'habitude de représenter un navire sous une effigie humaine existait aussi chez les Romains : sous la tête de Cléopâtre, on voit une petite proue, incorporée à la légende (fig. 16) ${ }^{1}$. Le symbole est ici parfaitement clair : il évoque la puissance navale.

Si l'on nous accorde cette interprétation de leur monnaie, on tiendra pour certain que les Parisii ont eu, bien avant la conquête romaine, le commerce fluvial pour activité essentielle.

Ainsi s'affirme, tout au long de son histoire, le rôle de Lutèce, port fluvial.

Dès l'époque gauloise, l'île de la Seine est le centre d'un peuple de navigateurs commerçants qui adopte le navire pour emblème de ses monnaies : l'existence d'un groupement professionnel est, dès ce moment, hautement vraisemblable. Au lendemain de la conquête, la corporation des nautes, favorisée par les nouveaux maîtres, est en plein essor. A la fin de ce premier siècle de vie romaine, un monument relatif à la navigation, que décore une proue de navire, s'élève dans l'ille dont la ville commence à sortir pour s'étaler sur la rive gauche. Là se construit, un ou deux siècles plus tard, tout près du fleuve, un vaste monument public, dû sans doute à la corporation d'armateurs qui domine la vie de la cité, et dont la décoration célèbre le commerce fluvial. Après les invasions, la ville, diminuée, rentre dans son ìle : mais, fortifiée, elle doit encore à sa position d'être le port d'attache de la flottille de la Seine, où les navires marchands qui firent sa fortune sont peut-être enrôlés... La nature avait dicté ce destin : Lutèce, dès ses débuts, avant même de devenir un nœud de routes romaines, fut le port central de la Gaule du nord ; elle le resta durant toute l'Antiquité.

\section{Paul-Marie Duval.}

(1) ConEx, 1, 1859, p. 37, nº 1, argent, frappée en Asie; au revers, à gauche de la tête d'Antoine, une tiare.

\section{ADDENDA}

P. 139, n. 1 : la base de colonne est reproduite dans F. Hoffbauer, Paris $\dot{a}$ travers les âyes, I, fasc. 1, 1882, Lutèce (par Albert LeNoIR), fig. 16, p. 13.

P. 141, 1. 25 : note : le décor en dents de scie de la coque se retrouve sur les barques des miroirs préhelléniques de Syros, J.-N. Svononos, Journ. intern. d'arch. num., XVI, 1914, fig. 7, p. 97; et, avec les points intercalés, sur la galère d'une monnaie de Carausius, Num. Chronicle, XVII, 1937, fig. 1-2, p. 306-307. 